\title{
Research on Anti-saturation Feedback Control Method for UAV Avionics System
}

\author{
Zhang Xiao-Jun ${ }^{1, a}$ \\ ${ }^{1}$ Huali College Guangdong University of Technology, 511325 Guangdong Guangzhou, China
}

\begin{abstract}
UAV avionics system is prone to saturation distortion under unsteady conditions, so anti-saturation control is needed. A control method of UAV avionics system based on anti-saturation feedback compensation is proposed. The anti-saturation control process of UAV avionics system is a multi-objective optimization process with multi-variables. The constrained parameter model of UAV avionics system control is constructed. Electromagnetic loss, torque, output power and other parameters are taken as constraint indexes, the original control information of UAV avionics system is treated with self-stabilization, the equivalent control circuit is designed, and the magnetic resonance transmission mode of avionics system is analyzed. An anti-saturation feedback tracking control method is used for steady-state control of the output voltage of the avionics system. The error compensation function is constructed to adjust the output adaptive parameters of the avionics system and the static anti-saturation compensator is constructed to compensate the power gain. The yaw error and the output steady-state error of the avionics system are reduced. The simulation results show that the proposed method has better output stability, lower output error, better real-time performance and better linear autodisturbance rejection control performance.
\end{abstract}

\section{INTRODUCTIONS}

Avionics system is called "Integrated avionics system", which is an important part of modern fighter and UAV. The control performance and output power of UAV avionics system are closely related to avionics system. It can be said that without a high-performance avionics system, there can be no high-performance UAV. UAV avionics system uses unified electrical control system and integrated circuit processor to realize the integrated control of aircraft and transmit relevant information between avionics via airborne data bus. The integrated avionics module is the center of UAV avionics system ${ }^{[1]}$. The integrated control module of avionics system includes DSP module and sensor module, motor module and course correction module. In each integrated avionics module, VHF navigation receiver, glide channel receiver and GPS receiver are integrated. The avionics system can effectively control the motor and electrical equipment of the UAV. During the control process of the UAV avionics system, it is affected by the error factors of the heading reference system and the magnetometer, and the saturation distortion is easy to occur. Anti-saturation feedback control is needed to improve the output control performance of UAV avionics system ${ }^{[2]}$.

At present, the control methods of UAV avionics system are mainly taken based on fuzzy neural network, fuzzy PID control motor control method, wavelet analysis based motor control method and so on ${ }^{[3]}$. With the above value method, the information of all kinds of avionics on the aircraft is processed uniformly, and the equipment with the same function or similar function is combined into one component to carry on the comprehensive control, and the control efficiency is obtained, among which, in reference [4], a control method of UAV avionics system based on electromagnetic coupling compensation feedback suppression is proposed. The constrained parameter model of UAV avionics system, such as voltage and current, is constructed, and information is exchanged by data bus and integrated avionics module. The optimal control of avionics system is realized, but the anti-saturation ability of this method is not good for avionics system. In reference [5], a coupling control algorithm for UAV avionics system based on voltage gain adaptive linear feedback compensation is proposed. The optimal control is taken based on the constrained parameter model of power loss and efficiency, and the output gain is improved by combining the particle swarm optimization algorithm, but the static saturation resistance of the algorithm is not good in the process of avionics system control.

The above problems need to be solved, a control method of UAV avionics system based on anti-saturation feedback compensation is proposed in this paper, and the constraint parameter model of UAV avionics system control is constructed. The original control information of

Corresponding author: ${ }^{\mathrm{a}} 271764735 @$ qq.com 
UAV avionics system is treated with self-stabilization, the equivalent control power is designed, and the magnetic resonance transmission mode of avionics system is analyzed. An anti-saturation feedback tracking control method is used for steady-state control of the output voltage of the avionics system. The error compensation function is constructed to adjust the output adaptive parameters of the avionics system and the static antisaturation compensator is constructed to compensate the power gain. The yaw error and the output steady-state error of the avionics system are reduced. Finally, the simulation experiments are carried out to demonstrate the superiority of this method in improving the anti-saturation feedback control ability of UAV avionics system.

\section{ANALYSIS OF CONTROL EQUIVALENT CIRCUIT OF AVIONICS SYSTEM AND DESCRIPTION OF CONTROLLED OBJECT}

\subsection{Analysis of control constraint parameters and equivalent circuit of output model for UAV avionics system}

The core control component of UAV avionics system is attitude and heading reference system. There are two integrated avionics modules, which LRU and display module are connected with the integrated avionics module ${ }^{[6]}$. All LRU exchange information through data bus and integrated avionics module. The display components in avionics system are mainly flight display (PFD) and multifunction display (MFD). The display module is installed directly on the display ${ }^{[7]}$. The UAV integrated avionics system uses vertical gyroscope to provide attitude signal and azimuth gyroscope and magnetic sensor to provide heading signal. In the overall structure of avionics system, there are two integrated avionics modules, which are connected with each LRU and display module. All LRU exchange information through data bus and integrated avionics module. The integrated avionics module uses a high speed data bus to match and exchange data with the corresponding display ${ }^{[8]}$. The display components in the integrated avionics system are mainly flight display PFD and MFD. The display module is directly installed on the display. The attitude and course reference system includes advanced sensors (including accelerometers and rate sensors) and connects magnetometers to obtain Earth's magnetic field information. The attitude sensor of avionics system is connected to the atmospheric data computer to obtain atmospheric data information. The attitude and course reference system provides the attitude and course information of the aircraft and communicates with the display and integrated avionics components through the ARINC 429 protocol. According to the above design principle, the equivalent circuit of UAV avionics system is designed to realize the efficient transmission of avionics system. The secondary resonance of UAV avionics system has the function of "SS" compensation. The equivalent circuit model of electric power transmission of UAV avionics system is constructed by using " $T$ " equivalent as shown in figure 1.

On the basis of the topological structure of the electric power transmission system of UAV avionics system, the parameter analysis of the controlled object and the construction of the control objective function are carried out. In the control design of avionics system, the whole integrated avionics system provides air pressure altitude, space velocity, vertical velocity and external atmospheric information, and ARINC 429 data interface is used to communicate with the main integrated avionics module, display, attitude and heading reference system ${ }^{[9]}$.

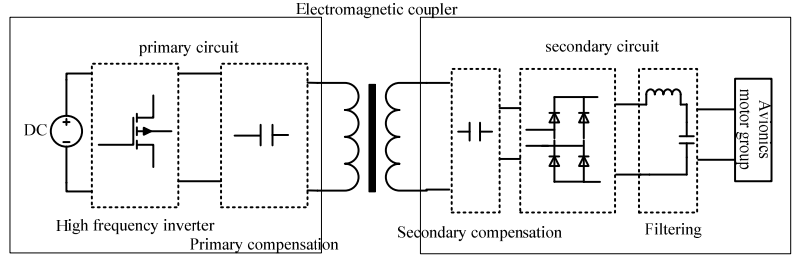

Fig. 1 Equivalent circuit model of UAV avionics system

The expression of control constraint geometric parameters and objective function of avionics system is established. The optimized parameters include polar logarithm $P$, pole arc coefficient $\beta$, magnetic pole thickness $l_{m}$, r winding thickness $J_{c u}$, air gap length $l_{g}$, rotor radius $r_{r}$, current density $\lambda=d_{b} / l_{s}$, conductor diameter $S$.

Define $A_{w}=\pi l_{w}\left(2 r_{r}+2 l_{g}+l_{w}\right)$. In most control methods, only a portion of the winding passes through the current at a given moment, as:

$$
N I=\pi l_{w}\left(2 r_{r}+2 l+l_{w}\right) J_{c u} k_{f} k_{c}
$$

The $k_{c}$ is a correction coefficient determined by the control system, which represents the ratio of the coils actually running in a certain state to the total coils (such as $k_{c}=2 / 3$ ). Ignoring armature reaction, the flux density on the surface of winding is:

$$
B_{g}=\frac{F_{m}}{A_{g} \Re}
$$

Where, $F_{m}$ is every pole magnetic EMF, $A_{g}$ is winding air gap area, and is $\mathfrak{R}$ winding.

\subsection{Description of control object for Avionics system}

According to the analysis of the control constraint parameter model of UAV avionics system, under the condition of steady state error, the robust control method based on disturbance observer can be used to estimate the control constraint parameter model as follows:

$$
\begin{gathered}
F_{m}=\frac{B_{r} l_{m}}{\mu_{0} \mu_{r 1}} \\
A_{g}=l_{s} \frac{\beta \pi}{p}\left(r_{r}+l_{g}\right)
\end{gathered}
$$




$$
\Re=\frac{1}{\mu_{0} l_{s} \beta \pi / p}\left(\frac{1}{\mu_{r 1}} \int_{r_{r}-l_{m}}^{r_{r}} \frac{d_{r}}{r}+\int_{r_{r}}^{r_{r}+l_{g}} \frac{d_{r}}{r}+\frac{1}{\mu_{r 2}} \int_{r_{r}+l_{g}}^{r_{r}+l_{g}+l_{w}} \frac{d_{r}}{r}\right)
$$

Where, the vacuum permeability of the electromagnetic coupler in the avionics system $\mu_{0}=4 \pi \times 10^{-7} \mathrm{H} / \mathrm{m}, \quad \mu_{r 1}$ and $\mu_{r 2}$ are permeability of permanent magnets and windings.

The switching circuit and resonant circuit can improve the transmission efficiency of the system, but the nonsinusoidal component in the current waveform leads to additional $\mathrm{AC} \operatorname{loss}^{[10]}$. In addition, with the parameters of electromagnetic loss, torque and output power as the constraint index, the effective impedance of the primary and secondary sides can be expressed as follows:

$$
\begin{gathered}
R=R_{d c}+\frac{\psi}{3} \Delta^{4} R_{d c}\left(\frac{I^{\prime}}{\omega \cdot I}\right) \\
\psi=\frac{5 p^{2}-1}{15} \\
\Delta=\frac{d_{e f f}}{\delta_{0}} \\
R_{d c}=4 \rho \frac{N \cdot M L T}{\pi \cdot S t r \cdot d_{c}^{2}} \\
\delta_{0}=\sqrt{\frac{1}{\pi f_{s w} \mu_{0} \delta}}
\end{gathered}
$$

Where, $\omega=2 \pi f$. Considering the input saturation error of UAV avionics system as uncertainty, the antisaturation compensation is carried out for the whole UAV avionics system. The calculation accuracy of leakage inductance and excitation inductance directly affects the resonance performance. The anti-saturation error tracking control method is used to optimize the avionics system.

\section{OPTIMIZATION OF CONTROLLER FOR UAV AVIONICS SYSTEM}

\subsection{Anti-saturation feedback tracking control}

On the basis of constructing the constraint parameter model of UAV avionics system control and analyzing the controlled object, the optimal design of UAV avionics system controller is carried out. This paper presents a control method for UAV avionics system based on antisaturation feedback compensation. The original control information of UAV avionics system is treated with selfstabilization, and the load balancing control of avionics system is carried out. The rectifier and filter circuit of avionics system can be approximately equivalent to a loop shaping, which is described as follows:

$$
R_{\mathrm{e} q}=8 V_{0}^{2} /\left(\pi^{2} P_{L}\right)
$$

The electromagnetic winding $C_{p}$ and capacitance $C_{s}$ are calculated, the continuous compensator leakage inductance and the working frequency of the avionics system meet the following requirements:

$$
\omega L_{l p}-\frac{1}{\omega C_{p}}=0 \Rightarrow C_{p}=\frac{1}{\omega^{2} L_{l p}}
$$

$$
\omega L_{l s}-\frac{1}{\omega C_{s}}=0 \Rightarrow C_{s}=\frac{1}{\omega^{2} L_{l s}}
$$

The electromagnetic torque of DC motor in avionics system is analyzed ${ }^{[11]}$. Combined with electromagnetic coupling compensation control method, the impedance of UAV avionics system is obtained as follows:

$$
\begin{gathered}
Z_{3}=R_{e q}+Z_{s} \\
Z_{2}=\frac{Z_{m}\left(R_{e q}+Z_{s}\right)}{Z_{m}+R_{e q}+Z_{s}} \\
Z_{1}=\frac{Z_{m}+R_{e q}+Z_{s}}{Z_{p}\left(Z_{m}+R_{e q}+Z_{s}\right)+\left(R_{e q}+Z_{s}\right) Z_{m}}
\end{gathered}
$$

Wherein

$$
\begin{gathered}
Z_{p}=R_{p}+j X_{p}=R_{p}+j\left(\omega L_{l p}-\frac{1}{\omega C_{p}}\right) \\
Z_{s}=R_{s}+j X_{s}=R_{s}+j\left(\omega L_{l s}-\frac{1}{\omega C_{s}}\right) \\
Z_{m}=j \omega L_{m}
\end{gathered}
$$

By analyzing the controller model above, it can be seen that the anti-saturation control process of avionics system is a multivariable multi-objective optimization process, and the adaptive feedback tracking control law is used to control the electrical automation of avionics system. The adaptive control optimization model of UAV avionics system is constructed to improve the output stability of UAV avionics system ${ }^{[12]}$.

\subsection{Static anti-saturation compensator}

The anti-saturation feedback tracking control method is used to control the output voltage steady-state of the avionics system. The voltage gain of the avionics system is calculated and the voltage gain is mainly determined by the load and frequency. The load determines the current flowing through the two sides of the electromagnetic coupler, and the frequency determines the impedance value of leakage inductance, excitation inductance and compensation capacitance. Because of the high frequency of the system, to reduce the on-loss, the rectifier circuit is constructed by using MUR1620CT ultra-fast recovery diode. The voltage gain is:

$$
G_{V}=\left|\frac{R_{e q}}{Z_{3}} \frac{Z_{2}}{Z_{1}}\right|=\left[(a c-b d)^{2}+(b c+a d)^{2}\right]^{-\frac{1}{2}}
$$

In the design of the anti-saturation compensator, the asymptotic stability of the reference model is taken into consideration, the saturation tracking error of the output voltage of the avionics system is obtained as follows:

$$
\frac{R_{e q}}{Z_{3}}=\frac{R_{\mathrm{e} q}}{R_{e q}+Z_{s}}
$$

In order to reduce the harmonics and prevent the magnetic saturation, the following harmonic rectifier is used to suppress the inertial error tracking compensation by the admittance controller: 


$$
\frac{Z_{2}}{Z_{1}}=\frac{Z_{m}\left(R_{e q}+Z_{s}\right)}{Z_{p}\left(R_{e q}+Z_{m}+Z_{s}\right)+Z_{m}\left(R_{e q}+Z_{s}\right)}
$$

Wherein

$$
\begin{gathered}
a=1+\frac{R_{s}}{R_{e q}} \\
b=\frac{X_{s}}{R_{e q}} \\
c=1+\frac{R_{p} R_{m}+X_{p} X_{m}}{R_{m}^{2}+X_{m}^{2}}+\frac{R_{p}\left(R_{e q}+R_{s}\right)+X_{p} X_{s}}{\left(R_{e q}+R_{s}\right)^{2}+X_{s}^{2}} \\
d=\frac{R_{m} X_{p}-R_{p} X_{m}}{R_{m}^{2}+X_{m}^{2}}+\frac{X_{p}\left(R_{e q}+R_{s}\right)-R_{p} X_{m}}{\left(R_{e q}+R_{s}\right)^{2}+X_{s}^{2}}
\end{gathered}
$$

An anti-saturation feedback tracking control method is used to control the output voltage of the avionics system $^{[13]}$. Considering the flux leakage coefficient $k_{1}$ and the span coefficient of the actual winding at the pole, the torque can be expressed as follows:

$$
\begin{gathered}
T_{e m}=\frac{\pi k_{f} k_{c} k_{1} k_{\beta} B_{r} l_{m} l_{s} l_{w}\left(2 r_{r}+2 l_{g}+l_{w}\right) J_{c u}}{\ln \left(\frac{r_{r}+l_{g}+l_{w}}{r_{r}-l_{m}}\right)} \\
k_{1}=1-\frac{1}{0.9\left[r_{r} /\left(\beta p\left(l_{g}+l_{w}\right)\right)\right]^{2}+1} \\
k_{\beta}=\frac{\alpha\left(\beta, k_{c}\right)}{k_{c}}
\end{gathered}
$$

Finally, the output power loss of avionics system is obtained as follows:

$$
P_{\text {loss }}=I_{P}^{2}\left(R_{p}+R_{c p}+2 R_{I G B T}\right)+I_{s}^{2}\left(R_{s}+R_{c s}+2 R_{d o n}\right)
$$

The transmission efficiency of UAV avionics system can be calculated by loss:

$$
\eta=\frac{I_{s}^{2} R_{e q}}{P_{\text {loss }}+I_{s}^{2} R_{e q}}
$$

Above all, the static anti-saturation compensator is used to compensate the power gain, which reduces the yaw error and the output steady-state error of the avionics system, and improves the output efficiency and gain.

\section{SIMULATION EXPERIMENT AND CONTROL PERFORMANCE ANALYSIS}

In order to test the application performance of this method in the optimal control of UAV avionics system, the simulation experiment is carried out. The experiment is designed with Matlab 7. The electromagnetic torque output of the avionics system is 10 N.m. the axial deviation $P_{x}=0.96 k_{x}^{\prime \prime} B_{\max }^{1.5} f^{1.5} \quad$, permeability $\mu_{0}=4 \pi \times 10^{-7} \mathrm{H} / \mathrm{m}$, open-loop gain is $12 \mathrm{~dB}$, closedloop gain is $22 \mathrm{~dB}$, initial transmission efficiency is 0.87 , and the control convergence threshold of static saturation error is 0.67 , other variable parameter designs are shown in Table 1
According to the above parameters, the control simulation of UAV avionics system is carried out, and the electromagnetic coupling magnetic field distribution of $\mathrm{UAV}$ avionics system is analyzed by using finite element analysis method as shown in figure 2 .

Table 1 Parameter setting

\begin{tabular}{cc}
\hline Motor control parameters & Value \\
\hline$N_{p}, N_{s}$ & 13.76 \\
$f / \mathrm{kHz})$ & 26.65 \\
$C_{p}, C_{s} / \mu \mathrm{F}$ & 0.345 \\
$V_{i} / \mathrm{V}$ & 61.32 \\
$V_{o} / \mathrm{V}$ & 25.98 \\
$R_{p}, R_{s}$ & $0.243,0.321$ \\
\hline
\end{tabular}

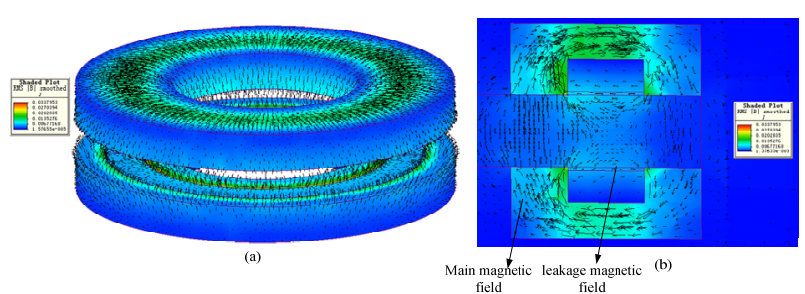

Fig. 2 Magnetic field distribution of electromagnetic coupling of UAV avionics system

The analysis figure 2 shows that the magnetic field equalization of anti-saturation feedback control of UAV avionics system using this method is good, which shows that the anti-saturation control ability is stronger. In order to further analyze the performance of the control algorithm in this paper, the output saturation error is taken as the test index, and the comparison results are obtained as shown in figure 3 .

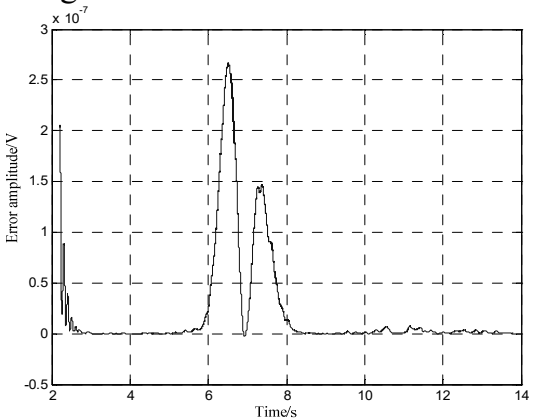

(a) Literature [4] method

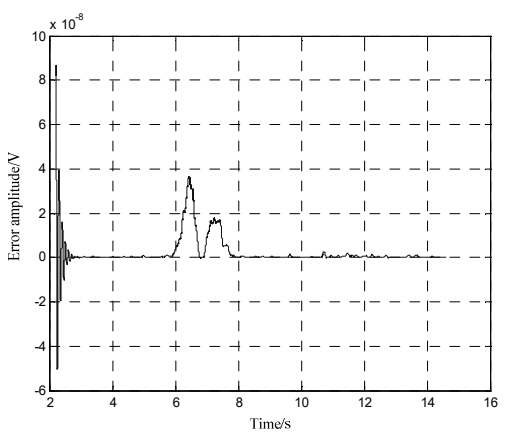

(b) Proposed method

Fig. 4 Convergence curve of the control output error 
Figure 4 shows that the anti-saturation feedback control of UAV avionics system using this method has better output control performance, lower output error and stronger anti-jamming ability. The time cost of different methods for control is compared as shown in figure 5 .

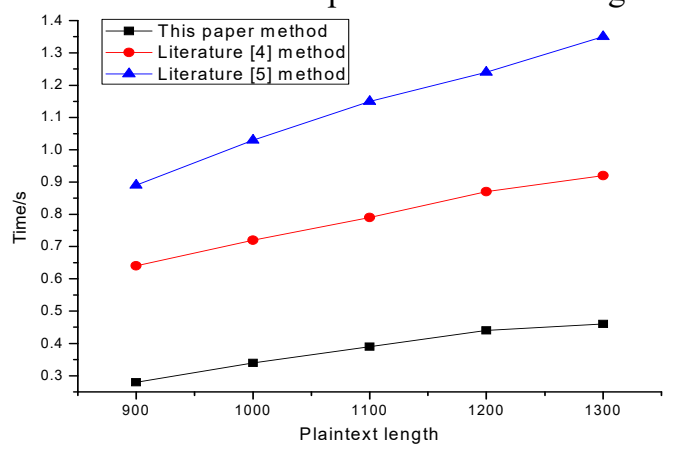

Fig.5 Comparison of time cost

The analysis figure 5 shows that the time cost of this method is shorter than traditional method, which can effectively improve the real-time performance of UAV avionics system control.

\section{CONCLUSIONS}

In the control process of UAV avionics system, due to the error factors of heading reference system and magnetometer, saturation distortion is easy to occur, so anti-saturation feedback control is needed to improve the output control performance of UAV avionics system. In this paper, a control method of UAV avionics system based on anti-saturation feedback compensation is proposed. The anti-saturation control process of UAV avionics system is a multi-objective optimization process with multi-variables. The equivalent control circuit is designed and the magnetic resonance transmission mode of avionics system is analyzed. The error compensation function is constructed to adjust the output adaptive parameters of the avionics system and the static antisaturation compensator is constructed to compensate the power gain. The yaw error and the output steady-state error of the avionics system are reduced. The results show that the proposed method has good output stability, low output error and good real-time performance. It has good performance in linear autoimmunity control of avionics system and has good application value.

\section{ACKNOWLEDGEMENTS}

This article is supported by the key platform and research projects of Guangdong provincial education department in 2015 (item No. 2015GXJK185).

\section{REFERENCES}

1. LU Xinghua Stability Control Aircraft Inertial Navigation System Design in Embedded Linux Environment[J]. Foreign Electronic Measurement Technology,2016,35(9):110-115.
2. LIU Kai, ZHU Ji-hong, YU Bo. Longitudinal control of aircraft with thrust vectoring using robust dynamic inversion [J]. Control and Decision, 2013, 28(7): 1113-1116.

3. Lu Xinghua, Zhan Shiwei, Yu Wenquan.Heading Correction Control Algorithm for UAV in Steepest Glide Trajectory [J] .Computer \& Digital Engineering, 2016,44(12): 2370-2374,2425.

4. GU Mingjiang, HU Guangbo. Research on Fault Diagnosis Method and System Construction of Pump Motor Power Supply Module[J]. Ship Electronic Engineering, 2018,38(2):119-123.

5. LI Ke,MI Jie. Research on Mechanical and Electrical Control Algorithm of Bionic Robot Based on Variable Structure PID [J].Journal of Henan University of Engineering(Natural Science Edition), 2016,28(2):32-37.

6. LU Xinghua. Anti Interference Control Algorithm for UAV Based on Attitude Fusion Filter[J]. Transducer and Microsystem Technologies, 2016,35(7):116-119.

7. JING Fengshui, YANG Chao, YANG Guodong, TAN Min. Robot Trajectory Rectification Control Methods. ROBOT, 2017, 39(3): 292-297.

8. Muhammad J, Altun H, Abo-Serie E. Welding seam profiling techniques based on active vision sensing for intelligent robotic welding[J]. International Journal of Advanced Manufacturing Technology, 2016, 88(1-4): 127-145.

9. DING Qing-qing,LU Wei,XU Chang-bo,et al. Passivity-based control of a three-phase shunt hybrid active power filter[J]. Journal of electrical machines and control,2014,18(05):1-6.

10. HUANG Wen-qing,ZHANG Xing-chun,ZHANG You-tong. Inverter dead time compensation method based on half PWM period control[J]. Journal of electrical machines and control,2014,18(05):24-29.

11. DENG Z H, CAO L B, JIANG Y Z, et al. Minimax probability TSK fuzzy system classifier: A more transparent and highly interpretable classification model[J]. IEEE Transactions on Fuzzy Systems, 2015, 23(4): 813-826.

12. Wan Z, Kothare M V. Efficient scheduled stabilizing output feedback model predictive control for constrained nonlinear systems[J]. IEEE Trans on Automatic Control, 2004, 49(7): 1172-1177.

13. Li K Y. PID Tuning for Optimal Closed-Loop Performance With Specified Gain and Phase Margins [J].IEEE Transactions on Control Systems Technology, 2013,21(3):1024-1030. 